TRANSACTIONS OF THE

AMERICAN MATHEMATICAL SOCIETY

Volume 354, Number 11, Pages 4585-4595

S 0002-9947(02)03052-0

Article electronically published on June 3, 2002

\title{
3-CONNECTED PLANAR SPACES UNIQUELY EMBED IN THE SPHERE
}

\author{
R. BRUCE RICHTER AND CARSTEN THOMASSEN
}

\begin{abstract}
We characterize those locally connected subsets of the sphere that have a unique embedding in the sphere - i.e., those for which every homeomorphism of the subset to itself extends to a homeomorphism of the sphere. This implies that if $\bar{G}$ is the closure of an embedding of a 3-connected graph in the sphere such that every 1-way infinite path in $G$ has a unique accumulation point in $\bar{G}$, then $\bar{G}$ has a unique embedding in the sphere. In particular, the standard (or Freudenthal) compactification of a 3-connected planar graph embeds uniquely in the sphere.
\end{abstract}

\section{Introduction}

One of the fundamental facts about finite planar graphs is that 3-connected planar graphs have unique embeddings in the sphere. In [4, Mohar asks how this theorem extends to infinite graphs and he proves that if the 3-connected planar graph $G$ is locally finite and all the faces are bounded by cycles, then $G$ has a unique embedding in the sphere. Imrich [3] proved that in any two embeddings of a 3 -connected graph in the sphere, the rotations at the vertices are the same in both embeddings.

In this work, we shall focus on pointed embeddings of locally finite graphs, i.e., embeddings in the sphere in which every 1-way infinite path has a unique accumulation point (which is not a point of the embedded graph). We will see in Section 6 that this implies every accumulation point arises this way. All graphs in this paper are at most countable and locally finite, i.e., every vertex has finite degree.

A compactification $\bar{G}$ of a graph $G$ is also pointed if every 1-way infinite path in $G$ converges to a unique accumulation point, that is, a point in $\bar{G} \backslash G$.

In other words, we are considering embeddings of the graph in which each end converges to some accumulation point, but two ends may converge to the same point; thus, we are simply identifying points of the end space. In the standard (or Freudenthal) compactification of a graph $G$, every end converges to a different point. Our work implies that if $G$ is a locally finite 3 -connected planar graph, then the Freudenthal compactification has a unique embedding in the sphere.

Our main result is the following.

Theorem 1. Let $G$ be a 3-connected, planar graph, and let $\bar{G}$ be a pointed compactification of $G$. Let $\phi_{1}, \phi_{2}: \bar{G} \rightarrow S^{2}$ be two embeddings of $\bar{G}$ in the sphere $S^{2}$. Then there is a homeomorphism $h: S^{2} \rightarrow S^{2}$ such that $\phi_{2}=h \circ \phi_{1}$.

Received by the editors October 23, 2001.

2000 Mathematics Subject Classification. Primary 57M15; Secondary 05C10, 57M20.

The first author acknowledges the financial support of NSERC.

(C) 2002 American Mathematical Society 
In fact, we shall prove something quite a bit more general than Theorem $1 \mathrm{~A}$ topological space is 2-connected if it is connected and remains so upon the deletion of any point. It is essentially 3-connected if it is 2-connected and the deletion of any two points results in one of: (i) a connected set; (ii) a set with two components, one of which is an open arc; (iii) three components, each of which is an open arc. This extends to topological spaces the concept of a 3-connected graph.

One of the main ingredients in the proof of Theorem 1 is the following, which is of independent interest. (In a private communication, Tom Tucker wondered if it were true that "3-connected" subsets of the sphere might uniquely embed in the sphere. This is what provoked us to consider this more general question.)

Theorem 2. Let $K$ be a compact, locally connected, 2-connected Hausdorff space. Suppose $\phi_{1}: K \rightarrow S^{2}$ is an embedding of $K$ in the sphere. Then $K$ is essentially 3connected if and only if for every embedding $\phi: K \rightarrow S^{2}$, there is a homeomorphism $h: S^{2} \rightarrow S^{2}$ such that $\phi=h \circ \phi_{1}$.

In Section 2 we give an example of a 3-connected (in fact it is infinitely connected) compact Hausdorff space that has two different embeddings in the sphere. It shows that some condition on $K$ is required for the conclusion of Theorem 2; it is natural to assume the space is locally connected.

In Section 3 we prove the following fact, which we use for the proof of Theorem 2 and seems to be of independent interest. It is a generalization of the fact that every 2 -connected finite graph has every face bounded by a cycle of the graph. If $K$ is a compact subset of the sphere, then a face of $K$ is a component of the complement of $K$.

Proposition 3. Let $K$ be a compact 2-connected locally connected subset of the sphere. Then every face of $K$ is bounded by a simple closed curve.

In Section 4, we give an example of a 3-connected graph $G$ embedded in the sphere so that the compactification (closure) is not locally connected and some face is not bounded by a simple closed curve.

Section 5 contains the proof of Theorem 2. In order to deduce Theorem 1 from Theorem 2, we need to prove that $\bar{G}$ is locally connected. This is done in Section 6 , where we prove that if $G$ is a 2-connected locally finite graph and $\bar{G}$ is a pointed compactification of $G$, then $\bar{G}$ is locally connected.

In Section 7 we prove that the Freudenthal compactification of a 3-connected planar graph embeds in the sphere and, therefore, it has a unique such embedding. We also discuss embeddings in the sphere with as few accumulation points as possible. If that number is finite, then the embedding is unique as well.

Since this work is aimed at both graph theorists and topologists, the proofs are as self-contained as possible.

\section{A 3-CONNECTED SPACE WITH TWO EMBEDDINGS}

Figure 1 shows a set $K$ which consists of the vertical line segment $L$ at the left (a dashed line in the figure) together with four sequences of rectangles "converging" to $L$. If we interchange the gray rectangles with the lower white ones, we obtain another embedding of $K$. So $K$ is not uniquely embeddable although the deletion of any finite point set leaves a connected space. Note that $K$ is not locally connected at any point of $L$. 


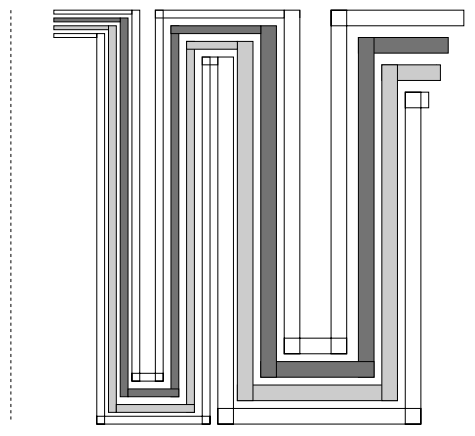

FIGURE 1. 3-connected, two embeddings

\section{Proof of Proposition 3}

In this section, we prove that the faces of a 2-connected locally connected compact subset of the sphere are all bounded by simple closed curves. Note that the example of the preceding section shows this is false without the assumption of local connection, even for 3-connected spaces. In the next section, we will exhibit another example, which has a dense 3-connected locally connected subset.

The following is an immediate consequence of Carathéodory's Theorem and its corollary as in [2].

Lemma 4. Let $K$ be a compact, connected, locally connected subset of $S^{2}$ and let $U$ be a face of $K$. Then $\partial U$ is connected and locally connected.

In order to prove Proposition 3, we shall first prove the following fact.

Lemma 5. Let $K$ be a compact, connected, locally connected subset of $S^{2}$ with more than one point. Then exactly one of the following holds:

(1) $K$ is an arc;

(2) $K$ is a simple closed curve;

(3) $K$ contains a point which is the common endpoint of three otherwise disjoint arcs.

Proof. Suppose neither (2) nor (3) holds.

Let $k$ be a point of $K$ that is an interior point of some arc $A^{\prime}$, with ends $a$ and $b$, contained in $K$. Then $k$ is an interior point of another arc $A$ which itself is contained in the interior of $A^{\prime}$. It is straightforward to use the facts that (2) and (3) do not hold and that $K$ is locally connected to deduce that the interior of $A$ is open in $K$.

Similarly, if $k$ is a point of $K$ that is not in the interior of any $\operatorname{arc}$ in $K$, then there is a half-open arc containing $k$, closed at $k$ and open in $K$.

It follows that these open sets provide an open cover of the compact set $K$; so they have a finite subcover. Let $A_{1}, \ldots, A_{k}$ be the closures of these finitely many open intervals. It is an easy induction to show that the union of these closed intervals is an arc.

Proof of Proposition 3. Suppose not. By Lemma 5, there are two alternatives, which we shall exclude.

First suppose $\partial U$ is just an $\operatorname{arc} A$. Since $K$ is 2-connected and the deletion of any interior point of $A$ disconnects $A, K \neq A$. Therefore, there is a point $k \in K \backslash A$. 
Since $A$ does not disconnect the sphere, there is an arc $B$ in $S^{2} \backslash A$ joining a point of $U$ with $k$. This arc must have a point of $\partial U$ in it; so there is a point $b \in \partial U \backslash A$, a contradiction.

Therefore, $\partial U$ must have a point $v$ that is a common end of three otherwise pairwise disjoint $\operatorname{arcs} A_{1}, A_{2}, A_{3}$ in $\partial U$. Since $K \backslash\{v\}$ is connected, there is an arc $B_{1} \subseteq K$ joining points in $A_{i}$ and $A_{j}$, for some $i \neq j$, such that $B_{1}$ is otherwise disjoint from $A_{1} \cup A_{2} \cup A_{3}$. Let $k \in\{1,2,3\} \backslash\{i, j\}$.

Now $A_{k}$ is a component of $\left(A_{1} \cup A_{2} \cup A_{3} \cup B_{1}\right) \backslash\{v\}$. Thus, there is an arc $B_{2}$ in $K$ joining a point of $A_{k} \backslash\{v\}$ to a point of $\left(A_{i} \cup A_{j} \cup B_{1}\right) \backslash\{v\}$ that is otherwise disjoint from $A_{1} \cup A_{2} \cup A_{3} \cup B_{1}$.

Let $\gamma_{1}$ be the simple closed curve in $A_{i} \cup A_{j} \cup B_{1}$. Then $\gamma_{1}$ separates the sphere into two discs, only one of which, say $\Delta$, contains $U$. The parts of $A_{i}$ and $A_{j}$ not in $\gamma_{1}$, together with $A_{k}$ and $B_{2}$ are all in $\Delta$. There is an arc $A$ contained in $\left(A_{k} \cup B_{2}\right) \cup\left(\left(A_{i} \cup A_{j}\right) \backslash \gamma_{1}\right)$ joining $v$ to some other point of $\gamma_{1}$. But this arc separates $\Delta$ into two parts, only one of which contains $U$. The other is incident with $v$ and a portion of one of $A_{i}$ and $A_{j}$, showing that portion is not in the boundary of $U$, a contradiction.

Hence $\partial U$ is a simple closed curve $\gamma$.

\section{Another Example}

Figure 2 shows an example of a 3-connected graph with an embedding in the sphere which has a face that is not bounded by a simple closed curve. Despite the fact that our proof does not extend to such a general situation, we wonder if the following might be true.

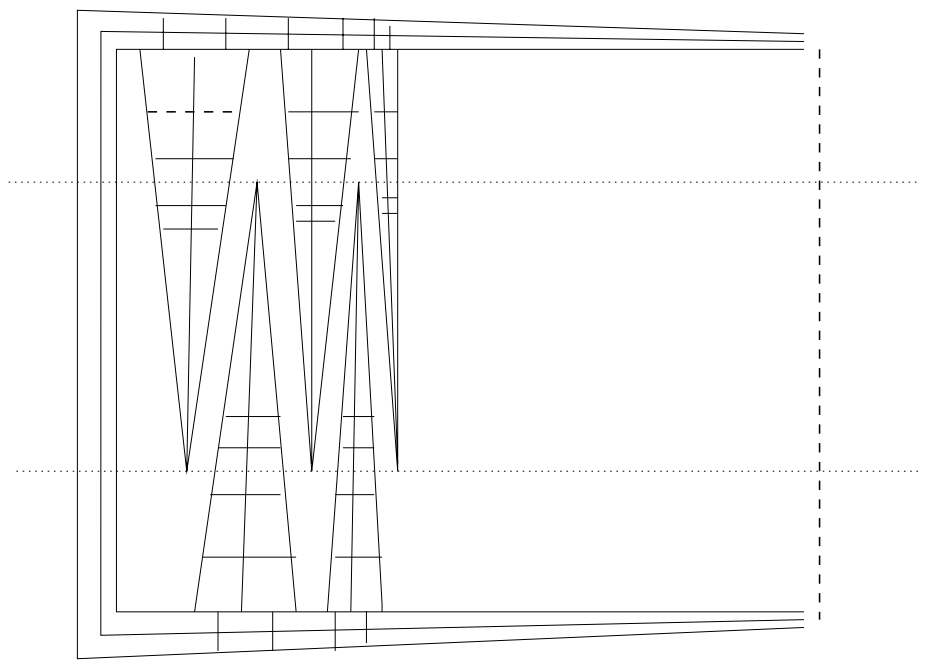

FiguRE 2. 3-connected, face not bounded by a cycle

Question. Let $G$ be a 3-connected graph embedded in the sphere with closure $\bar{G}$. Is it true that $\bar{G}$ is uniquely embeddable in the sphere?

The point here is that the infinite interior face is not bounded by a simple closed curve. For if it were bounded by the simple closed curve $\gamma$, say, then let $x$ be 
an interior point of the face and let $a$ be the midpoint of the right-hand vertical line of accumulation points. Considering the closed disc bounded by $\gamma$, there is a neighbourhood $N$ of $a$ and an $\operatorname{arc} A$ joining $x$ and $a$ such that $A \cap N$ is connected and $A$ is disjoint from the closure of the graph except for $a$.

But every small neighbourhood of $a$ is cut infinitely often by the spikes, and since the $\operatorname{arc} A$ must go around each spike, $A$ has infinitely many components in every small neighbourhood of $a$. Therefore, no such simple closed curve $\gamma$ exists.

We do not see how the example in Section 2 can be converted into the closure of a 3-connected graph embedded in the sphere.

\section{Proof of Theorem 2}

In this section we characterize those locally connected 2-connected compact subsets of the sphere that uniquely embed in the sphere as being those that are essentially 3 -connected.

Necessity in Theorem Q Suppose $K$ is 2-connected but not essentially 3 -connected. Then there is a pair $\{a, b\}$ of points in $K$ such that $K \backslash\{a, b\}$ is not connected and either has two components that are not open arcs or has at least three components, one of which is not an open arc, or has at least four components, all of which are open arcs. Since $\{a, b\}$ is closed in $K, K \backslash\{a, b\}$ is open; so every component is open. The closure $\bar{C}$ of any component $C$ of $K \backslash\{a, b\}$ is just $C \cup\{a, b\}$. It is arcwise connected and contains an arc from $a$ to $b$.

Let $P_{C}$ be an arc joining $a$ and $b$ in the closure of some other component of $K \backslash\{a, b\}$, so that $C \cup P_{C}$ is a 2-connected subset of $K$.

We assume first that some such $C$ is such that $\bar{C}$ is not just a single arc. Every face of $C \cup P_{C}$ is bounded by a simple closed curve; exactly two faces are incident with $P_{C}$. Let $P$ and $Q$ be the arcs complementary to $P_{C}$ in the boundaries of these faces, so that $P, Q$ and $P_{C}$ all have the same ends.

Note that $P \neq Q$ since $\bar{C}$ is not just an arc. It follows that $P \cup Q$ contains a simple closed curve $\gamma$, which we invert, keeping the two points of $P \cap Q$ fixed, to get a different embedding of $K$.

Otherwise, every $\bar{C}$ is just an arc. There are at least four, or else $K$ is essentially 3 -connected. It is now easy to interchange two adjacent ones to get different embeddings of $K$.

We shall prove the following version of the sufficiency in Theorem 2 .

Theorem 6. Let $K$ be a compact, locally connected, essentially 3-connected subset of the sphere. If $\phi: K \rightarrow S^{2}$ is an embedding of $K$ into the sphere, then there is a homeomorphism $h: S^{2} \rightarrow S^{2}$ such that $h(k)=\phi(k)$, for all $k \in K$.

Proof. We note that we can assume $K$ is not a simple closed curve, since then the result follows from the Jordan-Schönflies Theorem.

Let $\gamma$ be a simple closed curve bounding a face $U$ of $K$. We begin by showing that $K \backslash \gamma$ is connected.

To see this, let $C$ be a component of $K \backslash \gamma$. Call a point $x \in \gamma$ an attachment of $C$ if there is an $\operatorname{arc} A$ joining a point of $C$ to $x$ such that $A$ is otherwise disjoint from $\gamma$. Note that $A \backslash\{x\} \subseteq C$. 
Let $\operatorname{att}(C)$ denote the set of attachments of $C$. The fact that $K$ is 3-connected implies that either the closure of $C$ is an arc or $|\operatorname{att}(C)| \geq 3$. We may assume not every component of $K \backslash \gamma$ is an open arc whose closure is an arc, since otherwise either we are trivially done or $K$ is not essentially 3-connected.

Let $C$ and $C^{\prime}$ be distinct components of $K \backslash \gamma$, labelled so that the closure of $C^{\prime}$ is not just an arc. We note that if $w, x$ are attachments of $C$ and $y, z$ are attachments of $C^{\prime}$ such that $\{w, x\} \cap\{y, z\}=\emptyset$, then the four points $w, x, y, z$ occur in this cyclic order on $\gamma$. (Otherwise, we can add an arc in $U$ which, together with $\gamma$ and arcs in $C$ and $C^{\prime}$ form a $K_{3,3}$, a contradiction.)

It follows that if $w, x$ are fixed attachments of $C$, then all the attachments of $C^{\prime}$ occur in one of the two arcs in $\gamma$ having ends $w$ and $x$. Thus, there is a compact interval $I=[s, t]$ contained in $\gamma$ such that $\operatorname{att}\left(C^{\prime}\right) \subseteq I$ and $s, t$ are in the closure of $\operatorname{att}\left(C^{\prime}\right)$ in $\gamma$.

But now we claim that $K \backslash\{s, t\}$ is not connected. For let $c \in C$ and $c^{\prime} \in C^{\prime}$. If $K \backslash\{s, t\}$ is connected, there is an $\operatorname{arc} A$ joining $c$ and $c^{\prime}$ that avoids $s$ and $t$. There must be a subarc $A^{\prime}$ of $A$ joining points in each of the arcs of $\gamma \backslash\{s, t\}$, but then there is another component of $K \backslash \gamma$ that has attachments that overlap two from $C^{\prime}$ (one near $s$ and one near $t$ ), a contradiction.

So $K \backslash \gamma$ is connected.

Since $K \neq \gamma, \phi(\gamma)$ bounds a unique face of $\phi(K)$. Use the Jordan-Schönflies Theorem to extend the map $\phi$ into the interiors of these common faces.

For $x \in K$, set $h(x)=\phi(x)$. For $x \notin K$, let $F$ be the face of $K$ containing $x$ and let $\gamma$ be the boundary of $F$. We have an extension of $\phi$ from the interior of $F$ to the corresponding face of $\phi(\gamma)$. Set $h(x)$ to be the image of $x$ in that extension. Clearly, $h: S^{2} \rightarrow S^{2}$ is a bijection.

We now prove $h$ is continuous. This is trivial for $x \notin K$, and so we assume $x \in K$. For $x \in S^{2}$, let $N$ be a neighbourhood of $h(x)$. Since $\phi$ is continuous, there is a neighbourhood $M_{K}$ of $x$ in $K$ such that $\phi\left(M_{K}\right) \subseteq N$. Therefore, there is a neighbourhood $M$ of $x$ in $S^{2}$ such that $\phi(M \cap K) \subseteq N$. So there is an $\varepsilon>0$ such that $\phi(B(x ; \varepsilon) \cap K) \subseteq N$.

Let $\delta>0$ be such that $B(h(x) ; 4 \delta) \subseteq N$. For $n=1,2,3$, let $\gamma_{n}$ denote the circle with centre $h(x)$ and radius $n \delta$.

For each face $F$ intersecting $B(h(x) ; \delta)$ but not contained in $N$, there is an arc $B_{F}$ joining a point on $\gamma_{1}$ to a point on $\gamma_{3}$, but otherwise disjoint from $\gamma_{1} \cup \gamma_{3}$.

If there are infinitely many such $F$, then the $B_{F}$ have an accumulation point $z$ on the circle with centre $h(x)$ and radius $2 \delta$. We claim $K$ cannot be locally connected at $z$.

To see this, let $\delta>\alpha>0$ and consider the neighbourhood $V=B(z ; \alpha)$ of $z$. Infinitely many of the arcs $B_{F}$ go through $V$. Each such $B_{F}$ contains an arc $B_{F}^{\prime}$ contained in the closure of $V$ and having just its ends in the boundary of $V$. There are infinitely many such $B_{F}^{\prime}$ (each of which is disjoint from $K$ ), and between any two of them, there is a point of $K$, which cannot be reached in $V$ from $z$. This shows that no subneighbourhood of $V \cap K$ can be connected.

Thus, only finitely many faces of $\phi(K)$ can intersect $B(h(x) ; \delta)$ and not be contained in $M$. For each such face, we can reduce $\varepsilon$ to ensure that $h(B(x ; \varepsilon)) \cap F \subseteq M$. Since we only have to reduce $\varepsilon$ finitely often, we eventually obtain an $\varepsilon>0$ so that $h(B(x ; \varepsilon)) \subseteq M$, as required. 


\section{Pointed COMPACTIFICATIONS ARE LOCALLY CONNECTED}

In order to apply our results to compactifications of graphs, we clearly need to know which compactifications are locally connected. This seems to be an interesting problem in its own right.

We have the following natural sufficient condition, and it is the purpose of this section to prove this fact.

Theorem 7. Let $G$ be a 2-connected locally finite graph and let $\bar{G}$ be a pointed compactification of $G$. Then $\bar{G}$ is locally connected.

We shall make use of the following observation.

Proposition 8. Let $G$ be a 2-connected locally finite graph and let $\mathcal{P}$ be an infinite set of pairwise disjoint finite paths in $G$. Let $A$ and $B$ be sets of vertices such that every path in $\mathcal{P}$ has one end in $A$ and one end in $B$. Then $G$ has a 1-way infinite path containing infinitely many vertices in $A$ and infinitely many vertices in B. Moreover, if infinitely many of the paths in $\mathcal{P}$ are just edges, then the 1-way infinite path can be taken to use infinitely many of these edges.

Proposition 8 implies Theorem 7 . Let $x \in \bar{G}$. If $x \in G$, then it is easy to find arbitrarily small connected neighbourhoods of $x$. If $x \in \bar{G} \backslash G$, let $U$ be any neighbourhood of $x$ in $\bar{G}$ and let $V$ be another neighbourhood of $x$ such that the closure of $V$ is contained in $U$. (Since $\bar{G}$ is a compact Hausdorff space there is such a $V$.)

We shall show that there is a finite subset $W$ of $V(G)$ such that the union of all components of $G-W$ containing rays converging to $x$ are contained in $U$. We begin by proving that there is at least one ray that converges to $x$. If not, then there is a sequence of edges that contains $x$ as an accumulation point. Since $G$ is locally finite, we can assume the edges are pairwise disjoint. By the "moreover" in Proposition 8 there is a 1 -way infinite path $P$ containing infinitely many of the given edges. Since $x$ is an accumulation point of $P$, it must be the only one.

If no such set exists, let $W \subseteq V(G)$ be finite. Then $G-W$ contains a component $C_{W}$ with a 1-way infinite path $P_{W}$ that converges to $x$, but $P_{W}$ is not contained in $U$. Let $u_{W}$ denote a vertex of $P_{W}$ that is outside $U$, let $v_{W}$ denote a vertex of $P_{W}$ that is in $V$, and let $Q_{W}$ denote the path in $P_{W}$ joining $u_{W}$ and $v_{W}$.

Now define an infinite sequence $W_{0} \subseteq W_{1} \subseteq W_{2} \subseteq \ldots$ of finite subsets of $V(G)$ by $W_{0}=\emptyset$ and $W_{i+1}=W_{i} \cup V\left(Q_{W_{i}}\right)$. The paths $Q_{W_{i}}, i=0,1,2, \ldots$ are obviously pairwise disjoint.

Now apply Proposition 8 to the paths $Q_{W_{i}}$. This yields a 1-way infinite path $P$ in $G$ that has infinitely many $u_{W_{i}}$ and infinitely many $v_{W_{i}}$. The $u_{W_{i}}$ are contained in the compact set $\bar{G} \backslash U$; so they have a limit point $u \in \bar{G} \backslash U$. The $v_{W_{i}}$ are all contained in the compact set $\bar{V}$, and so they have a limit point $v \in \bar{V}$. Since $\bar{V} \subseteq U$, $u \neq v$. But then $P$ has two accumulation points, contradicting the hypothesis.

Therefore, there is a finite set $W$ of vertices such that the union of the components of $G-W$ containing rays converging to $x$ is contained in $U$. These sets, together with the usual open neighbourhoods for points in $G$ provide a basis for a topology $\mathcal{T}$ of the same point set $\bar{G}$. The topology which came with $\bar{G}$ has all its open sets unions of open sets in $\mathcal{T}$. Since $\bar{G}$ is, in both cases, compact and Hausdorff, the two topologies are the same. Therefore, every point of $\bar{G} \backslash G$ has arbitrarily small connected neighbourhoods. 
What remains is to prove Proposition [8. We begin with the following simple observation.

Lemma 9. Let $G$ be a 2-connected (possibly infinite, not necessarily locally finite) graph and let $H$ be a connected subgraph of $G$. Let $u, v, w$ be three vertices in the same component of $G-V(H)$. Then there is a path $P$ in $G$ from u to v, through $w$, such that $P \cap H$ is either empty or connected.

We use this to prove the following.

Lemma 10. Let $G$ be a 2-connected locally finite graph, let $H$ be a finite connected subgraph of $G$, let $R$ be a 1-way infinite path in $G$, let $A \subset V(G) \backslash V(R)$, and let $\mathcal{P}$ be an infinite set of pairwise disjoint paths in $G$, each with one end in $A$ and one end in $V(R)$. Then there is a path $P$ in $G-V(H)$ joining two vertices of $R$ and containing a vertex of $A$.

Proof. We can assume $R, A$ and the paths in $\mathcal{P}$ are all disjoint from $H$. Label the paths in $\mathcal{P}$ as $P_{1}, P_{2}, \ldots$, so that $P_{i}$ has ends $a_{i} \in A$ and $r_{i} \in V(R)$, with $r_{1}, r_{2}, r_{3}, \ldots$ appearing in this order in $R$.

For a non-negative integer $j$, suppose we have $j$ pairs of paths $\left\{Q_{1}, Q_{1}^{\prime}\right\}, \ldots$, $\left\{Q_{j}, Q_{j}^{\prime}\right\}$, such that, for each $k=1,2, \ldots, j$ : (i) $Q_{k}, Q_{k}^{\prime}$ join $a_{i_{k}} \in A$ to vertices of $R$; (ii) $Q_{k}, Q_{k}^{\prime}$ are disjoint except for $a_{i_{k}}$; (iii) $Q_{k}$ is disjoint from $H$; and (iv) $Q_{1}, Q_{2}, \ldots, Q_{j}$ are pairwise disjoint.

If some $Q_{k}^{\prime}$ is disjoint from $H$, the lemma is proved; so we can assume $Q_{k}^{\prime} \cap H$ is not empty. We show how to get one more pair of paths.

Let $H_{j}$ denote the union of $H$ with all the $Q_{k}, Q_{k}^{\prime}$ and the portion of $R$ from the origin to the last vertex of $R$ that is in any $Q_{k}$ or $Q_{k}^{\prime}$. Clearly $H_{j}$ is connected, there is an infinite tail $R_{j}$ of $R$ that is disjoint from $H_{j}$, and there is an infinite subset $\mathcal{P}_{j}$ of $\mathcal{P}$ that is disjoint from $H_{j}$. Let $A_{j}$ denote the subset of $A$ consisting of the ends of paths in $\mathcal{P}_{j}$.

Apply Lemma 9 to $H_{j}$, any $a \in A_{j}$, and any $r, r^{\prime} \in V\left(R_{j}\right)$. This gives the next pair of paths.

Thus, we have infinitely many such pairs $\left\{Q_{j}, Q_{j}^{\prime}\right\}$. As mentioned above, no $Q_{j}^{\prime}$ is disjoint from $H$. Let $Q_{j}^{\prime \prime}$ denote a maximal subpath of $Q_{j}^{\prime}$ that contains $a_{i_{j}}$ and is disjoint from $H$. If some $Q_{j}^{\prime \prime}$ intersects some $Q_{k}$, then the lemma follows immediately. So we can assume each $Q_{j}^{\prime \prime}$ is disjoint from each $Q_{k}$.

The other end of $Q_{j}^{\prime \prime}$ is adjacent to some vertex of $H$ and since there are only finitely many such vertices, some two $Q_{j}^{\prime \prime}$ have the same end. Thus, $Q_{j}^{\prime \prime}$ and $Q_{k}^{\prime \prime}$ intersect, for some $j \neq k$, and again it is easy to finish the proof.

It is now an easy matter to prove the following.

Lemma 11. Let $G$ be a 2-connected graph, let $R$ be a 1-way infinite path in $G$, let $A \subset V(G) \backslash V(R)$, and let $\mathcal{P}$ be an infinite set of pairwise disjoint paths in $G$, each with one end in $A$ and one end in $R$. Let $X$ be an infinite subset of $V(R)$. Then there is a 1-way infinite path in $G$ containing infinitely many elements of $A$ and infinitely many elements of $X$.

Proof. It is easy to use Lemma [10 to get an infinite sequence $Q_{1}, Q_{2}, \ldots$ of pairwise disjoint paths, each with just its ends in $R$, each containing a vertex of $A$, and the ends of $Q_{j}$ both occur in $R$ before either end of $Q_{j+1}$. (Apply Lemma 10 with $H_{j}$ the union of part of $R$ and the first $j$ of the $Q_{j}$ in order to get $Q_{j+1}$.) 
Now let $R^{\prime}$ be the 1-way infinite path obtained by traversing $R$ until a vertex of $X$ is reached. Then continue on $R$ to the next available $Q_{j}$, which we use in $R^{\prime}$. After $Q_{j}$, we traverse $R$ until the next vertex of $X$, then the next $Q_{j}$ and so on. Clearly, this yields a 1-way infinite path having infinitely many vertices from both $X$ and $A$.

We are now prepared to prove Proposition 8 .

Proof of Proposition 8 . We begin by finding a 1-way infinite path in $G$ that contains infinitely many vertices of $A$.

Let $T_{0}$ be any spanning tree of $G$ and let $x_{0}$ be any vertex of $T_{0}$. Given the tree $T_{i}$ and the vertex $x_{i}$ of $T_{i}$ such that $T_{i}$ contains infinitely many vertices of $A$, let $T_{i+1}$ be a component of $T_{i}-x_{i}$ that contains infinitely many vertices of $A$ and let $x_{i+1}$ be the vertex of $T_{i+1}$ adjacent in $T_{i}$ to $x_{i}$.

Clearly $x_{0}, x_{1}, x_{2}, \ldots$ are the vertices (in order) of a 1 -way infinite path $R_{0}$ in $G$. If $R_{0}$ has infinitely many vertices from $A$, then we are done. So assume not.

Each vertex of $A$ not in $R_{0}$ is joined in $T_{0}$ by a finite path to a vertex of $R_{0}$, which shows that for each vertex of $A$, there is an $i$ such that $a$ is not a vertex of $T_{j}$, for $j \geq i$. Thus, infinitely many paths from vertices of $A$ to vertices of $R_{0}$ are pairwise disjoint. Lemma 11 (with, say $X=V\left(R_{0}\right)$ ) yields a 1-way infinite path $R_{1}$ in $G$ containing infinitely many vertices from $A$.

If $R_{1}$ contains infinitely many vertices from $B$, then we are done; so assume not. By assumption, $\mathcal{P}$ has infinitely many disjoint paths joining vertices in $B$ to vertices in $R_{1}$. Lemma 11] (with $X=A \cap V\left(R_{1}\right)$ ) now yields the desired 1-way infinite path in $G$ containing infinitely many vertices from both $A$ and $B$.

\section{Particular cases}

Let $P$ and $Q$ be two 1-way infinite paths in a locally finite graph $G$. Define $P \sim Q$ to mean there are infinitely many pairwise disjoint paths joining vertices of $P$ to vertices of $Q$. It is straightforward (and well known, cf., 1]) to show that $\sim$ is an equivalence relation and the equivalence classes define the ends of $G$.

If $\bar{G}$ is a pointed compactification of $G$, then any two equivalent rays must have the same accumulation point (by Proposition 8). The Freudenthal compactification $\mathcal{F}(G)$ of $G$ is the compactification of $G$ such that any two ends have different accumulation points. The topology of $\mathcal{F}(G)$ is generated by the open subsets of $G$ together with the following open sets containing a limit point $x$ of an end $E$. For each finite subset $W$ of $V(G)$, the component of $G-W$ containing the 1-way infinite paths converging to $x$, together with all the other limit points of ends $E^{\prime}$ containing rays in that component, is the open set.

We note the following fact.

Lemma 12. Let $G$ be a locally finite 2-connected planar graph. Then $\mathcal{F}(G)$ embeds in the sphere.

Proof. Fix any embedding of $G$ in the sphere and let $G_{0}, G_{1}, \ldots$ be an ascending sequence of finite induced 2-connected subgraphs of $G$ such that $G=\bigcup_{n \geq 0} G_{n}$.

For each $n$, consider the embedding of $G_{n}$ contained in the embedding of $G$. There are at most finitely many components of $G-V\left(G_{n}\right)$. In particular, at most finitely many are contained in any particular face of $G_{n}$ and, since such a face is bounded by a simple closed curve, we may easily add a finite number of edges to $G_{n}$ so that no face contains two components of $G-V\left(G_{n}\right)$. 
We must be careful to add the edges so as to not have some vertex of $G$ obtain infinite degree, but this is easy, since every vertex is an attachment of some component of $G-V\left(G_{n}\right)$ for only finitely many $n$. We must also be careful to ensure that no end of $G$ converges to a point of $G$.

This latter can be guaranteed, because for each $x \in V(G) \cup E(G)$, there is an $n$ such that, if $x$ is a vertex, all edges of $G$ incident with $x$ are in $G_{n}$, or, if $x$ is an edge, all edges of $G$ incident with an end of $x$ are in $G_{n}$. In both cases, we can find an $\varepsilon>0$ (which depends only on $G_{n}$ ) to put an $\varepsilon$-neighbourhood around $x$ so that we forbid later intrusions into this neighbourhood. This ensures that no point of $G$ can be a limit point.

Since any two ends of $G$ are in different components of some finite subset of $V(G)$, eventually there is an $n$ so that the components of $G-V\left(G_{n}\right)$ containing the two ends are different, and these will be separated by a cycle, once we add the extra edges to $G_{n}$. Therefore, distinct ends converge to distinct points.

The following motivational result is now a simple consequence of all that has come before.

Theorem 13. Let $G$ be a 3-connected locally finite planar graph. Then $\mathcal{F}(G)$ has a unique embedding in the sphere.

Among all pointed compactifications of a graph $G, \mathcal{F}(G)$ has the most accumulation points, and every other one is obtained by identifying accumulation points of $\mathcal{F}(G)$.

At the other extreme, consider the compactification that embeds in the sphere with the fewest accumulation points. Let $\alpha(G)$ denote the smallest number of accumulation points, taken over all embeddings of $G$ in the sphere.

Theorem 14. Let $G$ be a 3-connected locally finite planar graph such that $\alpha(G)$ is finite. Then there is a unique compactification $\bar{G}$ of $G$ that embeds in the sphere and has $\alpha(G)$ accumulation points. Furthermore, $\bar{G}$ has a unique embedding in the sphere.

This theorem can be proved by proving a min-max theorem. For $G 3$-connected, locally finite and planar, let $H \subseteq G$ be a subdivision of a finite 3-connected graph. Let $q(H)$ be the number of faces of $H$ in which $G$ has infinitely many vertices. Then $\alpha(G)=\max q(H)$, where the maximum is taken over all finite subgraphs $H$ of $G$ that are subdivisions of 3-connected graphs.

Thus, the pointed compactifications of a locally finite 3-connected graph $G$ have the property that, of the ones that embed in the sphere, the ones with the most and fewest accumulation points are uniquely determined and they have unique embeddings. For a number $n$ of accumulation points between the smallest and the largest, there will typically be many different compactifications of $G$ having $n$ accumulation points. Each compactification that embeds in the sphere will, however, have a unique embedding there.

We conclude with a complete classification of those compact locally connected subsets $K$ of $S^{2}$ that have unique embeddings in $S^{2}$. If $K$ is 2-connected, then Theorem 2 implies the list is exactly the essentially 3-connected examples.

If $K$ is connected but not 2-connected, then $K$ has a point $x$ such that $K-\{x\}$ is not connected. If two or more of the components of $K-x$ are not half-open half-closed arcs, then we can find a simple closed curve $\gamma$ in $S^{2}$ that meets $K$ only 
at $x$ and separates these two components. By flipping one side of $\gamma$, we obtain a different embedding of $K$.

If $K-x$ has three or more components, and one of them is not just an arc, then we can separate the non-arc from at least two arcs, flip the non-arc side and get another embedding of $K$.

Finally, if $x$ is the end of at least four otherwise pairwise disjoint arcs, then we can easily get another embedding of $K$.

There is one more way we can get a second embedding of $K$ and that is if $x$ is incident with at least two faces of $K$.

Therefore, the connected but not 2-connected uniquely embeddable examples are: (i) a point; (ii) an arc; (iii) three arcs with a common end, but otherwise disjoint; (iv) any essentially 3-connected compact locally connected $L$ together with pairwise disjoint arcs, each having just an end in common with $L$, and each attachment point is incident with only one face of $L$.

Finally, we consider the not connected case. We note that $K$ has only finitely many components. If there are at least two components that are neither arcs nor points, then there are two embedddings. If $K$ consists only of pairwise disjoint arcs and points, then $K$ has a unique embedding. So we can assume $K$ has exactly one component $L$ that is neither an arc nor a point. The remaining components of $K$ are all arcs and points. If $L$ has more than one face and is not a simple closed curve, then Lemma 5 implies $K$ has at least two embeddings. If $L$ is a simple closed curve and there are more than two components, then $K$ has at least two embeddings. If $K$ is a simple closed curve plus either a point or an arc, then $K$ has a unique embedding. Finally, suppose $L$ has one face. The only possibilities that are not arcs and points are (iii) above and (iv), in which case the essentially 3-connected part is a disc.

\section{REFERENCES}

[1] R. Diestel, Decomposing infinite graphs, Disc. Math. 95 (1991), 69-89. MR 93b:05159

[2] A. Douady and J.H. Hubbard, Etude dynamique des polynomes complexes (Première Partie), Publications Mathématiques d'Orsay. MR 87f:58072a

[3] W. Imrich, On Whitney's Theorem on the unique embeddability of 3-connected graphs, in "Recent Advances in Graph Theory", Proc. 2nd Czechoslovak Sympos., Prague, 1974, 303306. MR 52:5462

[4] B. Mohar, Embeddings of infinite graphs, J. Combin. Theory, Ser. B 44 (1988), 29-43. MR 88k:05074

[5] C. Thomassen, The Jordan-Schönflies theorem and the classification of surfaces, Amer. Math. Monthly 99 (1992), 116-130. MR 92k:57026

Department of Combinatorics and Optimization, University of Waterloo, Waterloo, Ontario, Canada

E-mail address: brichter@math.uwaterloo.ca

Mathematical Institute, Technical University of Denmark, Lyngby, Denmark

E-mail address: c.thomassen@mat.dtu.dk 